ARTIGO ORIGINAL

Revista de Enfermagem do Centro-Oeste Mineiro

2021; 11/4162

DOI: http://dx.doi.org/10.19175/recom.v11i0.4162

www.ufsj.edu.br/recom

\title{
Conhecimentos, atitudes e práticas sobre aleitamento materno entre puérperas em alojamento conjunto
}

\author{
Knowledge, attitudes and practices about breastfeeding among postpartum women in rooming-in
Conocimientos, actitudes y prácticas sobre la lactancia materna entre personas en alojamiento conjunto

\section{RESUMO}

Objetivos: Avaliar o conhecimento, atitude e prática sobre aleitamento materno entre puérperas, em alojamento conjunto de uma maternidade com selo de Hospital Amigo da Criança e descrever os motivos do desmame precoce em gestações anteriores. Método: Inquérito com 157 puérperas em alojamento conjunto com análise descritiva e inferencial. Resultados: Faixa etária e paridade associaram-se ao conhecimento e à atitude. Mulheres acima de 26 anos (OR=0,672 [IC=0,564-0,801]) e multíparas (OR $=0,750[$ [IC $=0,661-0,851])$ têm mais chance de possuírem saberes adequados. A idade mais elevada ( $O R=0,356$ [IC $=0,172-0,735]$ ), planejamento da gravidez ( $O R=0,275$ [IC $=0,093-0,815])$ e multiparidade $(O R=0,475[I C=0,229-0,985])$ foram fatores de proteção à atitude. A consulta pré-natal associou-se à prática $(p=0,023)$ e enquanto fator de proteção $(\mathrm{OR}=0,313 \quad[\mathrm{IC}=0,112-0,873])$ ao aleitamento. Conclusão: A Enfermagem pode auxiliar positivamente no conhecimento, na atitude e na prática sobre aleitamento, auxiliando no cuidado preventivo do trauma mamilar como causa importante do desmame precoce.

Descritores: Conhecimentos; Atitudes e Prática em Saúde; Aleitamento Materno; Razão de Chances; Alojamento Conjunto; Enfermagem.

\section{ABSTRACT}

Purposes: To evaluate the knowledge, attitude, and practices related to breastfeeding among postpartum women in a maternity ward which holds the Child-Friendly Hospital seal (Selo Hospital Amigo da Criança) and to describe the reasons for early weaning in previous pregnancies. Method: A survey with 157 postpartum women at a joint maternity ward with descriptive and inferential analysis. Results: Age and parity were associated with knowledge and attitude. Women over $26(\mathrm{OR}=0.672[\mathrm{Cl}=0.564$ $0.801])$ and multiparous women $(\mathrm{OR}=0.750[\mathrm{Cl}=0.661-0.851])$ are more likely to have adequate knowledge. Higher ages $(\mathrm{OR}=0.356[\mathrm{Cl}=0.172-0.735])$, pregnancy planning $(\mathrm{OR}=0.275[\mathrm{Cl}=0.093-0.815])$ and multiparity $(\mathrm{OR}=0.475[\mathrm{Cl}=0.229-0.985])$ were associated to protection and attitude. Prenatal consultation was associated with practice $(p=0.023)$, while protective factor $(\mathrm{OR}=0.313 \quad[\mathrm{Cl}=0.112-0.873])$ was associated to breastfeeding. Conclusion: Nursing can be a positive assistance when it comes to knowledge, attitude, and practice about breastfeeding, helping in the preventive care of nipple trauma becoming an important cause of early weaning. Descriptors: Knowledge; Attitudes and Practice in Health; Breastfeeding; Odds Ratio; Joint Accommodation; Nursing.

\section{RESUMEN}

Objetivos: Evaluar el conocimiento, la actitud y la práctica de la lactancia materna entre las puérperas en una sala de maternidad con un sello del Hospital Amigo del Niño y describir las razones del destete temprano en embarazos anteriores. Método: Se trata de una encuesta con 157 mujeres puérperas en alojamiento conjunto con análisis descriptivo e inferencial. Resultados: El grupo de edad y la paridad se asociaron con el conocimiento y la actitud. Las mujeres mayores de 26 años $(\mathrm{OR}=0,672[\mathrm{Cl}=0,564-0,801])$ y las mujeres multiparosas $(\mathrm{OR}=0,750[\mathrm{Cl}=0,661$ $0,851])$ son más propensas a tener un conocimiento adecuado. La edad más alta (OR $=0.356[\mathrm{Cl}=0.172-0.735])$, la planificación del embarazo $(\mathrm{OR}=0.275[\mathrm{Cl}=0.093-0.815])$ y la multiparidad $(\mathrm{OR}=0.475[\mathrm{Cl}=0.229-0.985])$ fueron factores protectores a la actitud. La consulta prenatal se asoció con la práctica $(p=0,023)$ y como factor protector $(\mathrm{OR}=0,313[\mathrm{Cl}=0,112-0,873])$ con la lactancia materna. Conclusión: La enfermería puede ayudar positivamente en el conocimiento, la actitud y la práctica sobre la lactancia materna, ayudando en la atención preventiva del trauma mamilar, como una causa importante del destete temprano.

Descriptores: Conocimiento; Actitudes y Práctica en Salud; Lactancia Materna; Razón de Probabilidades; Alojamiento Conjunto; Enfermería.
Viviane Cordeiro de Queiroz $^{1}$
0 0000-0002-2037-921X

Smalyanna Sgren da Costa

Andrade $^{2}$

$\underline{0000-0002-9812-9376}$

Edna Samara Ribeiro César ${ }^{2}$
$0 \underline{0000-0002-1150-5157}$

Karen Krystine Gonçalves de Brito ${ }^{2}$ 0000-0002-2789-6957

Cintia Bezerra Almeida Costa ${ }^{1}$ 0000-0002-1179-5852

Simone Helena dos Santos Oliveira ${ }^{1}$ 0000-0002-9556-1403

${ }^{1}$ Universidade Federal da Paraíba, PB, Brasil.

${ }^{2}$ Faculdade de Enfermagem Nova Esperança, PB, Brasil.

Autor correspondente:

Viviane Cordeiro de Queiroz

E-mail: vivicordeiroqueiroz35@gmail.com

Como citar este artigo:

Queiroz VC, Andrade SSC, César, ESR, et al. Conhecimentos, atitudes e práticas sobre aleitamento materno entre puérperas em alojamento conjunto. Revista de Enfermagem do Centro-Oeste Mineiro. 2021;11:e4162. [Access___]; Available in:__. DOI: http://dx.doi.org/10.19175/recom.v11i0.4 $\underline{162}$ 


\section{INTRODUÇÃO}

A gestação pode ser definida como um fenômeno natural e transformador, causando diversas modificações no organismo materno, sejam de natureza física, para que ocorra uma adaptação à condição gravídica e puerperal, como também emocional, de modo a prepará-la para o momento do parto e à aceitação da nova identidade decorrente da maternidade ${ }^{(1)}$. Uma modificação do período puerperal para a vida da mulher é o aleitamento materno, que se constitui um importante marco nos primeiros meses de vida do bebê. Por meio dele, conseguem-se suprir todas as necessidades nutritivas do recémnascido, além de reforçar o aumento de anticorpos, ganho de peso, propiciar maior vínculo entre mãe e filho e promover o desenvolvimento das estruturas orais, que são responsáveis pelo funcionamento adequado do sistema estomatognático, que tem como funções a respiração, sucção, deglutição, mastigação e fala(2).

Sob esta ótica, a decisão da prática do aleitamento materno não é determinada apenas biologicamente, mas também emocional e socioculturalmente. Amamentar apenas ou não, bem como o desmame, tende a ser uma decisão tomada principalmente pela mãe. Essa escolha é influenciada por sua história de vida, rede de apoio, condições físicas e emocionais, assim como a situação de saúde do bebê e o valor social incutido à amamentação e à maternidade ${ }^{(3)}$.

No que se refere ao espaço hospitalar voltado ao incentivo da amamentação, surge o alojamento conjunto nas instituições, como forma de trazer mais integração entre a mãe e o bebê, contribuindo para estabelecer um relacionamento afetivo favorável desde o nascimento. Além disso, no alojamento conjunto, são implementadas orientações em saúde voltadas à família, permitindo o desenvolvimento de competências e habilidades, favorecendo a segurança emocional, quanto aos cuidados com o recém-nascido e possibilitando a redução da incidência de desmame precoce ${ }^{(4)}$. Além das vantagens e recomendações do aleitamento, nos primeiros seis meses de vida, determinadas situações especiais podem atrapalhar o estabelecimento do aleitamento materno exclusivo, entre elas: redução do nível de escolaridade, baixa renda e situação conjugal sem parceria. Entre outros fatores, a insegurança, baixa autoestima, o receio de não possuir leite suficiente, escassez de rede de apoio, sejam da família, companheiro, comunidade e/ou profissionais, imaturidade emocional e problemas de autoimagem, também, configuram-se elementos contribuintes à redução dos índices de aleitamento satisfatório ${ }^{(4)}$.

Entretanto, para que a iniciação e o estabelecimento do aleitamento materno tenham êxito, o desempenho do enfermeiro é considerado elemento relevante ao sucesso da amamentação e deve ser realizado desde o prénatal até o puerpério, seja no alojamento conjunto e/ou na atenção primária à saúde ${ }^{(5)}$. Verificando que o conhecimento da realidade das puérperas pode colaborar para a promoção da saúde materno-infantil e, por fim, compreendendo que o levantamento dos principais pontos relacionados às potencialidades e dificuldades no ato de amamentar constitui-se fator considerável à tomada de decisão clínica e assistencial do profissional de saúde, objetivou-se avaliar o conhecimento, a atitude e a prática sobre aleitamento materno entre puérperas, em alojamento conjunto de uma maternidade com selo de Hospital Amigo da Criança e descrever os motivos do desmame precoce em gestações anteriores.

\section{MÉTODO}

Trata-se de inquérito avaliativo e transversal do tipo conhecimento, atitude e prática, de abordagem quantitativa. A pesquisa do tipo "conhecimentos, atitudes e prática" é embasada no constructo da competência e habilidade, portanto não apresenta referencial teórico. A coleta foi realizada, no Alojamento Conjunto do Instituto Cândida Vargas, maternidade de referência ao atendimento de gestantes, puérperas e recém-nascidos e na Iniciativa Hospital Amigo da Criança no Estado (IHAC), localizado na cidade de João Pessoa, Paraíba, Brasil, realizada de julho a setembro de 2019, com puérperas internadas no alojamento conjunto.

Os critérios de seleção foram puérperas com maioridade etária, primíparas ou multíparas. Foram excluídas aquelas que não estavam aptas cognitiva ou emocionalmente (autorreferido), puérperas cujos filhos possuem malformação ou síndromes, puérperas mastectomizadas, contaminadas por HIV e Hepatite B que impossibilitavam a amamentação. A amostra foi calculada, por meio da Amostragem Aleatória Simples (AAS), para amostra finita, com população 
anual de 5.320 puérperas atendidas na maternidade. O nível de confiança foi de $95 \%$, com margem de erro de $5 \%$, ou seja, $\alpha=0,05$ ( $z=$ $1,96)$. A proporção foi de $12 \%(p=0.12)$, conforme indicadores da Organização Mundial da Saúde (OMS) para aleitamento materno exclusivo. A amostra probabilística foi calculada em 157 puérperas e não houve perdas.

As variáveis sociodemográficas foram idade, tempo de escolarização, ocupação, naturalidade, espaço geográfico, religião, etnia, rendimento mensal, carga horária, situação conjugal, consultas pré-natais, complementação láctea. Os aspectos sexuais e reprodutivos foram sexarca, uso de contraceptivos, via do parto, paridade, abortamento, infecções sexualmente transmissíveis e gestação planejada. Os hábitos de vida averiguados relacionaram-se ao fumo, etilismo e sedentarismo.

As fontes de informação relacionadas ao conhecimento sobre aleitamento materno exclusivo também foram avaliadas. 0 conhecimento, a atitude e a prática neste estudo foram determinados como: saberes, opiniões e condutas diante do objeto de estudo ${ }^{(6)}$. Para definir a satisfatoriedade dos construtos Conhecimento, Atitude e Prática (CAP), utilizaramse, como ponto de corte, acima de $70 \%$ dos critérios definidos para cada um, conforme adaptação de estudos sobre conhecimento ${ }^{(7)}$.

Em relação aos critérios de satisfatoriedade, foi considerado conhecimento satisfatório, quando a puérpera assinalou no mínimo: dois benefícios/vantagens para o binômio mãe-filho do aleitamento materno; dois cuidados em relação à pega correta; dois fatores que maximizam a produção do leite materno; mencionou que a amamentação exclusiva acontece por seis meses e o complemento lácteo depois deste período. E insatisfatório: quando pelo menos dois dos critérios elencados acima não foram atendidos pela puérpera e/ou não manifestasse opinião a respeito. A atitude foi satisfatória, quando citado: o desejo de amamentar o bebê; que a amamentação é imprescindível ao bebê saudável até seis meses de idade; complementação após seis meses; e que a amamentação exclusiva é sempre necessária ao bebê saudável. E insatisfatória, quando a puérpera não atendeu a pelo menos um dos critérios supracitados e/ou não teve opinião a respeito.

Para classificar a prática como satisfatória em multíparas, os critérios foram, quando a puérpera mencionou ter amamentado todos os filhos anteriores, de maneira exclusiva até os seis meses de idade do último filho, sem complemento anterior a esse período. Como prática insatisfatória em multíparas, quando ao menos um critério não foi atendido pela puérpera.

Para a coleta de dados, foi utilizado um formulário com 39 questões, construído para a pesquisa, com questões sobre caracterização sociodemográfica, reprodutiva, hábitos de vida e sobre conhecimento, atitude e prática das puérperas em alojamento conjunto sobre aleitamento materno. O conteúdo do constructo do CAP foi adaptado ${ }^{(6)}$.

O instrumento da pesquisa foi aplicado com as puérperas, em local adequado, na própria maternidade, assegurando sigilo e confidencialidade, em dias e horários préestabelecidos, nos turnos matutino e vespertino. O procedimento de coleta dos dados seguiu o plano de amostragem sistemática, com "salto" de um entre as participantes da pesquisa para atender ao critério probabilístico. Com o auxílio do Programa IBM Statistical Package for the Social Sciences (SPSS), versão 21, foi realizada a análise dos dados. Os resultados foram exibidos, contendo frequência absoluta e percentual, médias e desvio-padrão. Para a associação, utilizaram-se os Testes Quiquadrado e Exato de Fisher, com significância $\leq 0,05$.

Utilizaram-se a Razão de Chance lodds ratio) e o intervalo de confiança, que, por sua vez, sugeriu a chance ou a proteção gerada pelas variáveis selecionadas com significância para resultados e que não cruzou a nulidade, isto é, 1. Em relação ao valor da razão de chance, houve indicativo de risco, para resultados maiores que 1 (um), assim como aqueles menores que 1 (um) apontaram fator de proteção. A leitura aconteceu neste último, sob a forma de percentual, indicando maior chance ao desfecho positivo avaliado. O estudo foi aprovado pelo Comitê de Ética em Pesquisa da Faculdade de Enfermagem Nova Esperança, Parecer 3.389.184, Protocolo n. 51/2019, Certificado de Apresentação para Apreciação Ética n.15259319.4.0000.5179.

\section{RESULTADOS}

Em relação à caracterização sociodemográfica das puérperas, a idade média foi de 25,92 (DP $\pm 4,601)$, com 61 (38,9\%) mulheres entre $18-25$ anos e $96(61,1 \%) \geq 26$ anos 
de idade. Sobre a escolaridade, 90 (57,3\%) participantes possuíam até nove anos de escolarização e $67(42,7 \%)$, $\geq$ de 10 anos. Sobre a ocupação, $86(54,8 \%)$ puérperas não possuíam remuneração e 71 (45,2\%) possuíam ocupação remunerada. Quanto à naturalidade, 111 (70,7\%) mulheres eram procedentes da capital e 46 $(29,3 \%)$ de outros municípios do Estado, sendo $145(92,4 \%)$ da zona urbana e $11(7,0 \%)$ da zona rural. Quanto à religião, $82(52,2 \%)$ participantes eram católicas, 61 (38,9\%) evangélicas e 14 (8,9\%) tinham outras crenças religiosas. Sobre a etnia/cor autorreferida, 130 (82,8\%) puérperas eram pardas, $13(8,3 \%)$ brancas, sete $(4,5 \%)$ amarelas e sete $(4,5 \%)$ pretas. O rendimento mensal apontou $116(73,9 \%)$ mulheres vivendo com menos de um salário mínimo, 40 (25,5\%) entre 1-2 salários mínimos. A carga horária mostrou que $60(38,2 \%)$ trabalhavam mais de 40 horas semanais, $12(7,6 \%)$ de $30-40$ horas e duas $(1,3 \%)$ até 20 horas por semana. Por fim, 128 $(81,5 \%)$ mulheres viviam com parceiro e 29 $(18,5 \%)$ sem parceiro.

Sobre as características sexuais e reprodutivas das gestantes, a idade média da sexarca foi de 17,53 anos (DP $\pm 2,608$ ), com 48 $(30,6 \%)$ mulheres com sexarca até 15 anos e 109 $(69,4 \%) \geq 16$ anos. Em relação à gravidez indesejada, $142(90,4 \%)$ puérperas desejaram ter filho e 15 (9,6\%) não planejaram. Além disso, 98 $(62,4 \%)$ mulheres faziam uso de contraceptivo antes da gestação e 59 (37,6\%) não usavam qualquer método contraceptivo. Das que faziam uso, 50 (31,8\%) tomavam pílula, 29 (18,5\%) faziam uso do preservativo, $12 \quad(7,6 \%)$ anticoncepcional injetável, cinco (3,2\%) Dispositivo Intrauterino (DIU). O tipo de parto elencado por 121 (77,1\%) puérperas foi via vaginal e $36(22,9 \%)$ cirurgia cesariana. Sobre a paridade, 80 (51\%) eram primigestas e 77 (49\%) multigestas, $129(82,2 \%)$ nunca abortaram e 28 $(17,8 \%)$ sofreram abortamento, das quais 27 $(17,2 \%)$ tiveram apenas um episódio e uma (6\%) mais de um episódio de abortamento. Sobre a ocorrência de infecções sexualmente transmissíveis, 153 (97,5\%) não apresentaram e quatro $(2,5 \%)$ tiveram algum tipo de infecção sexual.

Em relação aos hábitos de vida, das 157 puérperas participantes do estudo, 153 (97,5\%) não eram fumantes, 145 (92,4\%) não etilistas e 96 $(61,1 \%)$ eram sedentárias. Das mulheres não sedentárias, 40 (66,6\%) praticavam atividade física de $1-2$ vezes na semana e 20 (33,3\%) praticavam 2-3 vezes na semana. Sobre o complemento dado no âmbito hospitalar, 147 $(93,6 \%)$ afirmaram que o bebê não recebeu fórmula láctea e $10(6,4 \%)$ disseram que seus bebês foram complementados no hospital. Sobre as consultas de atendimento pré-natal, $89(56,7 \%)$ realizaram mais de seis consultas e $68(43,3 \%)$ mulheres até cinco consultas.

Sobre as fontes de informação relacionadas ao conhecimento sobre amamentação, as mulheres elencaram 321 respostas, das quais 86 $(26,7 \%)$ foi família, $85(26,4 \%)$ profissionais de saúde, 37 (11,5\%) escola, 34 (10,5\%) amigos, 23 $(7,1 \%)$ cartazes e folders, $22(6,8 \%)$ internet, 19 $(5,9 \%)$ televisão, 15 (4,6\%) campanhas e palestras. $\mathrm{Na}$ Tabela 1, encontram-se os critérios relacionados aos conhecimentos sobre aleitamento materno entre as gestantes.

Sobre o conhecimento relacionado ao aleitamento materno, os benefícios como prover todos os nutrientes, contribuição quanto ao crescimento e desenvolvimento do bebê e emagrecimento materno foram os mais elencados. A abertura máxima da boca do bebê e o apoio corporal adequado foram os cuidados principais da pega e posicionamento. A ingestão de água, sucos e chás foram as estratégias mais citadas para o aumento da produção láctea. A maioria indicou o aleitamento materno exclusivo por um período mínimo de seis meses conforme a Tabela

\begin{tabular}{|c|c|c|}
\hline Conhecimentos & $f$ & $\%$ \\
\hline \multicolumn{3}{|l|}{ Benefícios $(\mathrm{N}=731)^{*}$} \\
\hline Todos os nutrientes necessários para o bebê & 123 & 16,8 \\
\hline Contribui para o desenvolvimento e crescimento do bebê & 106 & 14,5 \\
\hline Emagrecimento materno & 88 & 12,0 \\
\hline Servir como vacina natural para o bebê & 74 & 10,1 \\
\hline Redução de cólicas no bebê & 56 & 7,6 \\
\hline Reduz o risco de câncer de mama e de ovário & 54 & $\begin{array}{c}7,3 \\
\text { (Continua) }\end{array}$ \\
\hline
\end{tabular}


Tabela 1 - Conhecimentos sobre aleitamento materno das puérperas em alojamento conjunto. João Pessoa, PB, Brasil, 2019. ( $n=157)$

\begin{tabular}{|c|c|c|}
\hline Conhecimentos & $\mathrm{f}$ & $\%$ \\
\hline Melhoria de edemas & 51 & 6,9 \\
\hline Reduz o risco de depressão pós-parto & 45 & 6,1 \\
\hline Reduz o sangramento no pós-parto & 44 & 6,0 \\
\hline Diminuição de cólicas uterinas/menstruais & 34 & 4,6 \\
\hline Útero a retornar ao tamanho anterior à gravidez & 34 & 4,6 \\
\hline Maturação do trato gastrintestinal do bebê & 22 & 3 \\
\hline \multicolumn{3}{|l|}{ Cuidados com a pega $(N=474)^{*}$} \\
\hline A boca do bebê bem aberta & 114 & 24 \\
\hline Mais aréola visível acima da boca do bebê & 109 & 23 \\
\hline O queixo do bebê tocando a mama & 102 & 21,5 \\
\hline Abocanhar boa parte da aréola & 75 & 15,8 \\
\hline Bebê de frente para a mama, com o nariz na altura do mamilo & 74 & 15,6 \\
\hline \multicolumn{3}{|l|}{ Cuidados com o posicionamento $(N=395)^{*}$} \\
\hline O bebê deve estar bem apoiado & 99 & 25 \\
\hline Corpo do bebê próximo à mãe & 89 & 22,5 \\
\hline Bebê com cabeça e tronco bem alinhados & 78 & 19,7 \\
\hline Sentada/deitada e bem apoiada & 68 & 17,2 \\
\hline Bebê de frente para a mama com o nariz na altura do mamilo & 61 & 15,4 \\
\hline \multicolumn{3}{|l|}{ Maneiras de aumentar a produção $(N=414)^{*}$} \\
\hline Ingerir bastante água, sucos e chás & 115 & 27,7 \\
\hline Dieta equilibrada & 113 & 27,2 \\
\hline Dormir adequadamente & 101 & 24,4 \\
\hline Pega e posicionamento adequado & 85 & 20,5 \\
\hline \multicolumn{3}{|l|}{ Tempo que o bebê deve ser amamentado ( $N=157)$} \\
\hline Até 6 meses & 77 & 49 \\
\hline Após 6 meses & 57 & 36,3 \\
\hline Antes de 6 meses & 23 & 14,6 \\
\hline \multicolumn{3}{|l|}{ Alimentos para os primeiros seis meses de vida $(N=157)$} \\
\hline Somente leite materno & 118 & 75,2 \\
\hline Leite materno e mais & 30 & 19,1 \\
\hline Não tenho opinião & 9 & 5,7 \\
\hline \multicolumn{3}{|l|}{ Atitudes $(\mathrm{N}=157)$} \\
\hline \multicolumn{3}{|l|}{ Desejo em amamentar } \\
\hline Até os 6 meses & 78 & 49,7 \\
\hline Até os 24 meses & 77 & 49 \\
\hline Não deseja amamentar & 2 & 1,3 \\
\hline \multicolumn{3}{|l|}{ Leite materno complementado } \\
\hline Após seis meses de idade do bebê saudável & 115 & 73,2 \\
\hline Antes dos seis meses de idade do bebê saudável & 34 & 21,7 \\
\hline Em qualquer idade do bebê saudável & 6 & 3,8 \\
\hline Não tenho opinião & 2 & 1,3 \\
\hline \multicolumn{3}{|l|}{ Amamentação exclusiva } \\
\hline Sempre necessário & 140 & 89,2 \\
\hline Pouco necessário & 15 & 9,6 \\
\hline Desnecessário & 2 & 1,3 \\
\hline \multicolumn{3}{|l|}{ Práticas $(\mathrm{N}=77)^{\dagger}$} \\
\hline \multicolumn{3}{|l|}{ Amamentou anteriormente } \\
\hline Todos os seus filhos & 72 & 45,9 \\
\hline Nem todos os seus filhos & 3 & 1,9 \\
\hline Não sei/ Não quero responder & 2 & 1,3 \\
\hline \multicolumn{3}{|l|}{ Amamentação exclusiva } \\
\hline Até 6 meses de idade do bebê & 65 & 41,4 \\
\hline Após 6 meses de idade do bebê & 8 & 5,1 \\
\hline De 1 a 5 meses de idade do bebê & 4 & 2,5 \\
\hline \multicolumn{3}{|l|}{ Amamentação complementada } \\
\hline De 6 a 12 meses de idade do bebê & 58 & 36,9 \\
\hline Após 12 meses de idade do bebê & 11 & 7 \\
\hline De 1 a 5 meses de idade do bebê & 8 & 5,1 \\
\hline
\end{tabular}

*Variáveis de múltiplas respostas; ${ }^{\dagger}$ Multíparas.

Fonte: Elaborado pelos autores.

Sobre as atitudes, a maioria expressou o desejo de amamentar exclusivamente, acreditam que o aleitamento apresenta muitas vantagens e que a alimentação complementar deve ser dada, 
após os seis meses do bebê, cujo Aleitamento Materno Exclusivo (AME) é sempre necessário. Sobre a prática das multíparas dos motivos, para não amamentar exclusivamente, relatados pelas multíparas, 64 (32,4\%) relataram dor, 55 (27,9\%) traumas mamilares, 37 (18,7\%) ausência da rede de apoio, $20(10,1 \%)$ ingurgitamento, $19(9,6 \%)$ mastite e 2 (1\%) citaram leite insuficiente, sem desejo de amamentar e complicação materna pós-parto.

Em relação aos critérios de satisfatoriedade, 137 (87,3\%) puérperas tiveram conhecimento satisfatório e 20 (12,7\%), insatisfatório. Quanto à atitude, 115 (73,2\%) tiveram opinião satisfatória e 42 (26,8\%), insatisfatória. Em relação à prática, entre as 77 que já amamentaram, 55 (71,4\%) mulheres tiveram prática satisfatória e 22 (28,5\%), insatisfatória. Assim, neste estudo, o conhecimento, a atitude e a prática do aleitamento materno foram satisfatórios.
Conforme a Tabela 2, a faixa etária $(p<0,0001)$, a sexarca $(p<0,0001)$, episódio de abortamento $(p=0,025)$ e paridade $(p<0,0001)$ associaram-se estatisticamente ao conhecimento. As maiores prevalências apontaram conhecimento satisfatório, para mulheres com idade $\geq 26$ anos, com sexarca $\geq 16$ anos, sem episódio de abortamento e multíparas. Além disso, mulheres mais maduras (OR $=0,672$ [IC $=0,564-0,801])$, com sexarca, após 16 anos (OR=0,185 [IC=0,068-0,500]) e multíparas (OR=0,750 [IC=0,661-0,851]), possuem 67\%, 18\% e $75 \%$ mais chance de possuir saber satisfatório, sendo essas variáveis consideradas fatores de proteção ao aleitamento materno. Mulheres não brancas possuem 1,16 maior chance de possuir conhecimento insatisfatório $(\mathrm{OR}=1,161$ [IC $=1,088$ $1,240]$. O mesmo acontece para aquelas que não sofreram abortamento, com chance de 1,18 (OR=1,183 [IC=1,099-1,274]) maior.

Tabela 2 - Razão de chance e associação entre conhecimento sobre amamentação com variáveis sociodemográficas, sexual, reprodutiva e de atendimento. João Pessoa, PB, Brasil, 2019. ( $n=157)$

\begin{tabular}{|c|c|c|c|c|c|c|}
\hline \multirow[t]{2}{*}{ Variáveis } & \multicolumn{5}{|c|}{ Conhecimento } & \multirow[t]{3}{*}{$\mathrm{OR}^{\S}(\mathrm{IC}) \|$} \\
\hline & \multicolumn{2}{|c|}{ Satisfatório } & \multicolumn{2}{|c|}{ Insatisfatório } & \multirow[t]{2}{*}{$\mathrm{p}^{*}$} & \\
\hline Faixa etária & $f$ & $\%$ & $f$ & $\%$ & & \\
\hline $18-25$ anos & 41 & 26,1 & 20 & 12,7 & & \\
\hline$\geq 26$ anos & 96 & 61,1 & 0 & 0 & $<0,0001^{\ddagger}$ & $0,672(0,564-0,801)$ \\
\hline \multicolumn{7}{|l|}{ Escolaridade } \\
\hline Até 9 anos & 75 & 47,7 & 15 & 9,5 & & \\
\hline$\geq 10$ anos & 62 & 39,4 & 5 & 3,1 & $0,087^{\dagger}$ & $0,403(0,139-1,172)$ \\
\hline \multicolumn{7}{|l|}{ Ocupação } \\
\hline Não remunerada & 78 & 49,6 & 8 & 5,1 & \multirow[b]{2}{*}{$0,155^{+}$} & \multirow[b]{2}{*}{$0,504(0,194-1,312)$} \\
\hline Remunerada & 59 & 37,5 & 12 & 7,6 & & \\
\hline \multicolumn{7}{|l|}{ Religião (N=143) } \\
\hline Católica & 71 & 49,6 & 11 & 7,6 & \multirow[b]{2}{*}{$0,958^{+}$} & \multirow[b]{2}{*}{$0,974(0,366-2,590)$} \\
\hline Evangélica & 53 & 37 & 8 & 5,5 & & \\
\hline \multicolumn{7}{|l|}{ Cor } \\
\hline Branca & 13 & 2,5 & 0 & 0 & \multirow[b]{2}{*}{$0,376^{\ddagger}$} & \multirow[b]{2}{*}{$1,161(1,088-1,240)$} \\
\hline Não Branca & 124 & 84,7 & 20 & 12,7 & & \\
\hline \multicolumn{7}{|l|}{ Fumante } \\
\hline Sim & 4 & 8,1 & 0 & 0 & \multirow[b]{2}{*}{$1,000^{\ddagger}$} & \multirow[b]{2}{*}{$1,150(1,082-1,223)$} \\
\hline Não & 133 & 78,1 & 20 & 11,7 & & \\
\hline \multicolumn{7}{|l|}{ Etilista } \\
\hline Sim & 9 & 5,7 & 3 & 1,9 & \multirow[b]{2}{*}{$0,183^{\ddagger}$} & \multirow[b]{2}{*}{$0,398(0,098-1,618)$} \\
\hline Não & 128 & 81,5 & 17 & 10,8 & & \\
\hline \multicolumn{7}{|l|}{ Atividade Física } \\
\hline Sim & 52 & 33,1 & 9 & 5,7 & \multirow[b]{2}{*}{$0,546^{+}$} & \multirow[b]{2}{*}{$0,748(0,290-1,926)$} \\
\hline Não & 85 & 54,1 & 11 & 7,0 & & \\
\hline
\end{tabular}

(Continua) 
Tabela 2 - Razão de chance e associação entre conhecimento sobre amamentação com variáveis sociodemográficas, sexual, reprodutiva e de atendimento. João Pessoa, PB, Brasil, 2019. $(n=157)$

\begin{tabular}{|c|c|c|c|c|c|c|}
\hline \multirow[t]{2}{*}{ Variáveis } & \multicolumn{5}{|c|}{ Conhecimento } & \multirow[t]{2}{*}{$\mathrm{OR}^{\S}(\mathrm{IC}) \mathrm{II}$} \\
\hline & \multicolumn{2}{|c|}{ Satisfatório } & \multicolumn{2}{|c|}{ Insatisfatório } & $\mathrm{p}^{*}$ & \\
\hline Até 15 anos & 35 & 22,2 & 13 & 8,2 & & \\
\hline$\geq 16$ anos & 102 & 64,9 & 7 & 4,4 & $<0,0001^{\dagger}$ & $0,185(0,068-0,500)$ \\
\hline \multicolumn{7}{|l|}{ Conjugalidade } \\
\hline Com parceiro & 113 & 71,9 & 15 & 9,5 & \multirow[b]{2}{*}{$0,421^{+}$} & \\
\hline Sem parceiro & 24 & 15,2 & 5 & 3,1 & & $1,569(0,520-4,733)$ \\
\hline \multicolumn{7}{|l|}{ Gravidez planejada } \\
\hline Não & 12 & 7,6 & 3 & 1,9 & & \\
\hline Sim & 125 & 79,6 & 17 & 10,8 & $0,410^{\ddagger}$ & $0,544(0,139-2,126)$ \\
\hline \multicolumn{7}{|l|}{ Via de parto } \\
\hline Vaginal & 104 & 66,2 & 17 & 10,8 & & \\
\hline Cesariana & 33 & 21 & 3 & 1,9 & $0,569^{\ddagger}$ & $1,798(0,496-6,521)$ \\
\hline \multicolumn{7}{|l|}{ Abortamento } \\
\hline Sim & 21 & 14 & 0 & 0 & & \\
\hline Não & 109 & 72,6 & 20 & 13,3 & $0,025^{\ddagger}$ & $1,183(1,099-1,274)$ \\
\hline \multicolumn{7}{|l|}{ Paridade } \\
\hline Primíparas & 60 & 38,2 & 20 & 12,7 & & \\
\hline Multíparas & 77 & 49 & 0 & 0 & $<0,0001^{\ddagger}$ & $0,750(0,661-0,851)$ \\
\hline \multicolumn{7}{|l|}{ Consultas } \\
\hline Até 5 & 56 & 35,6 & 12 & 7,6 & & \\
\hline$\geq 6$ & 81 & 51,5 & 8 & 5,1 & $0,107^{\dagger}$ & $0,461(0,177-1,200)$ \\
\hline \multicolumn{7}{|l|}{ Complementação } \\
\hline Sim & 10 & 6,3 & 0 & 0 & & \\
\hline Não & 127 & 80,8 & 20 & 12,7 & $0,363^{\ddagger}$ & $1,157(1,086-1,234)$ \\
\hline
\end{tabular}

${ }^{*} \mathrm{p}=$ Significância estatística; ${ }^{\dagger}$ Valor de $\mathrm{p}$ obtido pelo Teste do Qui-quadrado; ${ }^{\ddagger}$ Valor de $\mathrm{p}$ obtido pelo Teste Exato de Fisher; ${ }^{\S} \mathrm{OR}=$ Razão de Chance; "IC = Intervalo de Confiança de 95\%.

Fonte: Elaborado pelos Autores.

Quanto às atitudes, houve associação estatística com a faixa etária $(p=0,004)$, gravidez planejada $(p=0,014)$ e paridade $(p=0,043)$, indicando prevalência maior para atitudes satisfatórias entre mulheres com idade $\geq 26$ anos, que planejaram a gravidez e multíparas. A idade mais elevada $(\mathrm{OR}=0,356 \quad[\mathrm{IC}=0,172-0,735])$, planejamento da gravidez $(\mathrm{OR}=0,275$ [IC $=0,093$ $0,815])$ e multiparidade (OR=0,475 [IC=0,2990,985]) apresentaram-se como fatores de proteção ao aleitamento materno, respectivamente, $35 \%, 27 \%$ e $47 \%$ com mais chance à atitude satisfatória, conforme a Tabela 3.

Tabela 3 - Razão de chance e associação entre a atitude sobre amamentação com variáveis sociodemográficas, sexual, reprodutiva e de atendimento. João Pessoa, PB, Brasil, 2019. ( $n=157)$

\begin{tabular}{|c|c|c|c|c|c|c|}
\hline \multirow[t]{2}{*}{ Variáveis } & \multicolumn{5}{|c|}{ Atitude } & \multirow[t]{3}{*}{$\mathrm{OR}^{\S}(\mathrm{IC}) \mathrm{II}$} \\
\hline & \multicolumn{2}{|c|}{ Satisfatório } & \multicolumn{2}{|c|}{ Insatisfatório } & \multirow[t]{2}{*}{$p^{*}$} & \\
\hline Faixa etária & $f$ & $\%$ & $f$ & $\%$ & & \\
\hline 18-25 anos & 37 & 23,5 & 24 & 15,4 & & \\
\hline$>26$ anos & 78 & 49,6 & 18 & 11,4 & $0,004^{+}$ & $0,356(0,172-0,735)$ \\
\hline \multicolumn{7}{|l|}{ Escolaridade } \\
\hline Até 9 anos & 65 & 41,4 & 25 & 15,9 & & \\
\hline$\geq 10$ anos & 50 & 31,8 & 17 & 10,8 & $0,736^{+}$ & $0,884(0,431-1,813)$ \\
\hline \multicolumn{7}{|l|}{ Ocupação } \\
\hline Não remunerada & 67 & 42,6 & 19 & 12,1 & & \\
\hline Remunerada & 48 & 30,5 & 23 & 14,6 & $0,147^{+}$ & $0,592(0,290-1,206)$ \\
\hline
\end{tabular}


8| Queiroz VC, Andrade SSC, César, ESR, et al.

Tabela 3 - Razão de chance e associação entre a atitude sobre amamentação com variáveis sociodemográficas, sexual, reprodutiva e de atendimento. João Pessoa, PB, Brasil, 2019. (n=157)

\begin{tabular}{|c|c|c|c|c|c|c|}
\hline \multirow[t]{2}{*}{ Variáveis } & \multicolumn{5}{|c|}{ Atitude } & \multirow[t]{2}{*}{$\mathrm{OR}^{\S}(\mathrm{IC}) \mathrm{II}$} \\
\hline & \multicolumn{2}{|c|}{ Satisfatório } & \multicolumn{2}{|c|}{ Insatisfatório } & \multirow[t]{2}{*}{$\mathrm{p}^{*}$} & \\
\hline \multicolumn{6}{|l|}{ Religião (N=143) } & \\
\hline Católica & 62 & 43,3 & 20 & 13,9 & \multirow[b]{2}{*}{$0,269^{+}$} & \multirow[b]{2}{*}{$1,512(0,725-3,153)$} \\
\hline Evangélica & 41 & 28,6 & 20 & 13,9 & & \\
\hline \multicolumn{7}{|l|}{ Cor } \\
\hline Branca & 12 & 7,6 & 1 & 0,6 & \multirow[b]{2}{*}{$0,187^{\ddagger}$} & \multirow[b]{2}{*}{$4,777(0,602-37,925)$} \\
\hline Não Branca & 103 & 65,6 & 41 & 26,1 & & \\
\hline \multicolumn{7}{|l|}{ Fumante } \\
\hline Sim & 2 & 1,2 & 2 & 1,2 & \multirow[b]{2}{*}{$0,290^{\ddagger}$} & \multirow[b]{2}{*}{$0,354(0,048-2,597)$} \\
\hline Não & 113 & 71,9 & 40 & 25,4 & & \\
\hline \multicolumn{7}{|l|}{ Etilista } \\
\hline Sim & 6 & 3,8 & 6 & 3,8 & & \\
\hline Não & 109 & 69,4 & 36 & 22,9 & $0,058^{+}$ & $0,330(0,100-1,089)$ \\
\hline \multicolumn{7}{|l|}{ Atividade Física } \\
\hline Sim & 42 & 26,7 & 19 & 12,1 & \multirow[b]{2}{*}{$0,321^{+}$} & \multirow[b]{2}{*}{$0,696(0,340-1,426)$} \\
\hline Não & 73 & 46,5 & 23 & 14,6 & & \\
\hline \multicolumn{7}{|l|}{ Sexarca } \\
\hline Até 15 anos & 35 & 22,2 & 13 & 8,2 & \multirow[b]{2}{*}{$0,950^{+}$} & \multirow[b]{2}{*}{$0,976(0,454-2,098)$} \\
\hline$\geq 16$ anos & 80 & 50,9 & 29 & 18,4 & & \\
\hline \multicolumn{7}{|l|}{ Conjugalidade } \\
\hline Com parceiro & 93 & 59,2 & 35 & 22,2 & \multirow[b]{2}{*}{$0,725^{+}$} & \multirow[b]{2}{*}{$0,845(0,332-2,154)$} \\
\hline Sem parceiro & 22 & 14 & 7 & 4,4 & & \\
\hline Gravidez planejada & & & & & & \\
\hline Não & 7 & 4,4 & 8 & 5,1 & & \\
\hline Sim & 108 & 68,7 & 34 & 21,6 & $0,014^{+}$ & $0,275(0,093-0,815)$ \\
\hline Via de parto & & & & & & \\
\hline Vaginal & 88 & 56 & 33 & 21 & & \\
\hline Cesariana & 27 & 17,2 & 9 & 5,7 & $0,787^{+}$ & $1,125(0,479-2,642)$ \\
\hline Abortamento & & & & & & \\
\hline Sim & 21 & 13,3 & 7 & 4,4 & & \\
\hline Não & 94 & 59,8 & 35 & 22,2 & $0,817^{+}$ & $1,117(0,437-2,858)$ \\
\hline Paridade & & & & & & \\
\hline Primíparas & 53 & 33,7 & 27 & 17,2 & & \\
\hline Multíparas & 62 & 39,4 & 15 & 9,5 & $0,043^{+}$ & $0,475(0,229-0,985)$ \\
\hline Consultas & & & & & & \\
\hline Até 5 & 46 & 29,3 & 22 & 14 & & \\
\hline$\geq 6$ & 69 & 43,9 & 20 & 12,7 & $0,166^{\dagger}$ & $0,606(0,298-1,234)$ \\
\hline Complementação & & & & & & \\
\hline Sim & 6 & 3,8 & 4 & 2,5 & & \\
\hline Não & 109 & 69,4 & 38 & 24,2 & $0,459^{\ddagger}$ & $0,523(0,140-1,954)$ \\
\hline
\end{tabular}

${ }^{\circ} \mathrm{p}=$ Significância estatística; ${ }^{\dagger}$ Valor de $\mathrm{p}$ obtido pelo Teste do Qui-quadrado; ${ }^{\ddagger}$ Valor de $\mathrm{p}$ obtido pelo Teste Exato de Fisher; ${ }^{\S} \mathrm{OR}=$ Razão de Chance; "IC = Intervalo de Confiança de 95\%.

Fonte: Elaborado pelos autores.

Sobre a prática, consultas de pré-natal demonstraram-se estatisticamente significativas $(p=0,023)$, indicando prevalência maior para adequabilidade entre mulheres com mais de seis atendimentos de pré-natal. Ainda, o maior número de consultas apresentou-se como fator de proteção à prática do aleitamento materno em
$31 \%(\mathrm{OR}=0,313$ [IC=0,112-0,873]). Mulheres não fumantes apresentaram 1,4 vezes (OR=1,415 [IC=1,223-1,637]) mais chance de amamentar, ao passo que planejar a gravidez aumenta 3,6 vezes (OR=3,619 [IC=2,515-5,207]) a chance da prática adequada (Tabela 4). 
Tabela 4 - Razão de chance e associação entre a prática sobre amamentação com variáveis sociodemográficas, sexual, reprodutiva e de atendimento. João Pessoa, PB, Brasil, 2019. (n=157)

\begin{tabular}{|c|c|c|c|c|c|c|}
\hline \multirow[t]{2}{*}{ Variáveis } & \multicolumn{5}{|c|}{ Prática } & \multirow[t]{2}{*}{$\mathrm{OR}^{\S}(\mathrm{IC}) \|$} \\
\hline & \multicolumn{2}{|c|}{ Satisfatório } & \multicolumn{2}{|c|}{ Insatisfatório } & \multirow[t]{2}{*}{$\mathrm{p}^{*}$} & \\
\hline Escolaridade & $f$ & $\%$ & $f$ & $\%$ & & \\
\hline Até 9anos & 25 & 32,4 & 9 & 11,6 & & \\
\hline$\geq 10$ anos & 30 & 38,9 & 13 & 16,8 & $0,717^{+}$ & $1,204(0,442-3,279)$ \\
\hline \multicolumn{7}{|l|}{ Ocupação } \\
\hline Não remunerada & 41 & 53,2 & 13 & 16,8 & \multirow[b]{2}{*}{$0,181^{+}$} & \multirow[b]{2}{*}{$0,493(0,174-1,401)$} \\
\hline Remunerada & 14 & 18,1 & 9 & 11,6 & & \\
\hline \multicolumn{7}{|l|}{ Religião (N=68) } \\
\hline Católica & 21 & 30,8 & 10 & 14,7 & \multirow[b]{2}{*}{$0,637^{+}$} & \multirow[b]{2}{*}{$0,778(0,273-2,213)$} \\
\hline Evangélica & 27 & 39,7 & 10 & 14,7 & & \\
\hline \multicolumn{7}{|l|}{ Cor } \\
\hline Branca & 4 & 5,1 & 5 & 6,4 & \multirow[b]{2}{*}{$1,000^{\ddagger}$} & \multirow[b]{2}{*}{$0,267(0,064-1,108)$} \\
\hline Não Branca & 51 & 66,2 & 17 & 22 & & \\
\hline \multicolumn{7}{|l|}{ Fumante } \\
\hline Sim & 2 & 2,6 & 0 & 0 & \multirow[b]{2}{*}{$0,601^{\ddagger}$} & \multirow[b]{2}{*}{$1,415(1,223-1,637)$} \\
\hline Não & 53 & 68,8 & 22 & 28,5 & & \\
\hline \multicolumn{7}{|l|}{ Etilista } \\
\hline Sim & 1 & 1,3 & 1 & 1,3 & & \\
\hline Não & 54 & 70,1 & 21 & 27,2 & $0,492^{\ddagger}$ & $0,389(0,023-6,507)$ \\
\hline \multicolumn{7}{|l|}{ Atividade Física } \\
\hline Sim & 16 & 20,7 & 9 & 11,6 & \multirow[b]{2}{*}{$0,317^{\dagger}$} & \\
\hline Não & 39 & 50,6 & 13 & 16,8 & & $0,593(0,212-1,660)$ \\
\hline \multicolumn{7}{|l|}{ Sexarca } \\
\hline Até 15 anos & 7 & 9 & 4 & 5,1 & & \\
\hline$\geq 16$ anos & 48 & 62,3 & 18 & 23,3 & $0,719^{\ddagger}$ & $0,656(0,171-2,513)$ \\
\hline \multicolumn{7}{|l|}{ Conjugalidade } \\
\hline Com parceiro & 52 & 67,5 & 20 & 25,9 & \multirow[b]{2}{*}{$0,620^{\ddagger}$} & \\
\hline Sem parceiro & 3 & 3,9 & 2 & 2,6 & & $1,733(0,269-11,157)$ \\
\hline Gravidez planejada & & & & & & \\
\hline Não & 0 & 0 & 1 & 1,3 & & \\
\hline Sim & 55 & 71,4 & 21 & 27,2 & $0,286^{\ddagger}$ & $3,619(2,515-5,207)$ \\
\hline Via de parto & & & & & & \\
\hline Vaginal & 37 & 48 & 13 & 16,8 & & \\
\hline Cesariana & 18 & 23,3 & 9 & 11,6 & $0,497^{+}$ & $0,703(0,253-1,948)$ \\
\hline Abortamento & & & & & & \\
\hline $\operatorname{sim}$ & 9 & 11,6 & 5 & 6,4 & & \\
\hline Não & 46 & 59,7 & 17 & 22 & $0,513^{+}$ & $0,665(0,195-2,268)$ \\
\hline Consultas & & & & & & \\
\hline Até 5 & 15 & 19,5 & 12 & 15,5 & & \\
\hline$\geq 6$ & 40 & 51,9 & 10 & 12,9 & $0,023^{+}$ & $0,313(0,112-0,873)$ \\
\hline Complementação & & & & & & \\
\hline Sim & 5 & 6,4 & 2 & 2,6 & & \\
\hline Não & 50 & 64,9 & 20 & 25,9 & $1,000^{\ddagger}$ & $1,000(0,179-5,584)$ \\
\hline
\end{tabular}

${ }^{*} \mathrm{p}=$ Significância estatística; ${ }^{\dagger}$ Valor de $\mathrm{p}$ obtido pelo Teste do Qui-quadrado; ${ }^{\ddagger}$ Valor de $\mathrm{p}$ obtido pelo Teste Exato de Fisher; ${ }^{\S} \mathrm{OR}=$ Razão de Chance; "IIC = Intervalo de Confiança de 95\%.

Fonte: Elaborado pelos autores.

\section{DISCUSSÃO}

A caracterização das mulheres, neste estudo, assemelha-se a outras pesquisas nacionais e internacionais, quanto ao perfil de usuárias entre 21 e 44 anos, com ensino médio completo, sem atividade laboral ${ }^{(8)}$, estabilidade da 
união, religião ortodoxa, escolarização e ocupação, embora a literatura aponte nuances de escolarização em nível superior e prevalência de mulheres desempregadas ${ }^{(9)}$.

Sobre as características sexuais, reprodutivas e hábitos de vida, estudo em São Paulo identificou gravidez planejada e sexarca semelhantes aos resultados desta pesquisa. $\mathrm{O}$ uso de métodos contraceptivos e a ampliação da informação podem ser um fator de segurança para jovens não engravidarem mais cedo e desejarem suas gestações ${ }^{(7)}$. Inquérito CAP, realizado na zona urbana de Dakar, verificou que o tipo de parto mais observado foi o vaginal ${ }^{(9)}$.

Em uma maternidade pública no Rio de Janeiro, grande parte das puérperas não eram fumantes/etilistas, embora existam localidades com mulheres fumantes nos estágios iniciais da gravidez ${ }^{(10)}$, o que não foi o caso desta amostra. Em relação às consultas de pré-natal, em Minas Gerais, puérperas realizaram o número adequado, conforme os programas governamentais com porcentagem maior entre as mais maduras ${ }^{(11)}$. 0 início precoce da assistência pré-natal é recomendado com, no mínimo, seis consultas de acompanhamento, sendo, preferencialmente, uma no primeiro trimestre, duas no segundo trimestre e três no terceiro trimestre da gestação $^{(12)}$.

$\mathrm{Na}$ amostra estudada, o número de consultas confluiu com as recomendações governamentais e sobre a prática. Mais de seis atendimentos apresentaram-se como fator de proteção à prática do AM. Neste contexto, a Enfermagem torna-se relevante, enquanto parte da equipe de saúde da atenção básica, no sentido de atuar de maneira precoce na captação das gestantes ainda no primeiro trimestre, a fim de favorecer a quantidade mínima de consultas recomendadas para um pré-natal de qualidade, inclusive, favorecendo o compartilhamento de saberes sobre aleitamento materno ainda durante a gestação. Sobre as fontes de informação, estudos internacionais apontaram o profissional de saúde ${ }^{(9-10,13-15)}$, além da mídia eletrônica(15). Entretanto outros apontam a família como a principal rede de apoio primária(13-14,16).

Nesta pesquisa, a família e profissionais de saúde foram as principais fontes de informação sobre aleitamento. A maneira com que a família define suas prioridades, bem como sua forma de olhar e valorizar a mulher e a criança, pode exercer influência positiva (ajuda) ou negativa (impedimento) neste processo de amamentação(15).

As orientações sobre aleitamento materno, durante a gestação, constituem-se elementos salutares à compreensão da sua importância. Não obstante, pesquisa evidenciou que, embora as mães tivessem recebido informações sobre o aleitamento dos profissionais de saúde, a maioria não sabia que o aleitamento materno exclusivo protege o bebê contra diarreia(10). Em outro estudo, o conhecimento estimulou o comportamento, quando, na Etiópia, 88,8\% das mães praticavam o aleitamento materno exclusivo, nos primeiros seis meses de vida, concluindo que as informações compartilhadas às mães pela equipe de saúde influenciaram positivamente a adesão(16).

Na Nigéria, as puérperas sabiam que o AME é até os seis meses de vida, mas apontaram que apenas $o$ bebê era beneficiado pela amamentação, com vantagem relacionada à nutrição adequada para o lactente. Menos da metade sabia sobre os benefícios imunológicos e do vínculo biopsicossocial mãe/bebê(17). Já no Quênia, investigação com 400 mães mostrou que a maioria afirmou que o leite materno é suficiente até os seis primeiros meses de vida e que apenas após se deve introduzir alimentos complementares $^{(18)}$. Esses dados estão em conformidade com os resultados supracitados neste estudo.

Em Gana, resultado de pesquisa expôs que $34 \%$ das mães complementaram com água e $22 \%$ das mães relataram que o leite materno não é capaz de suprir as necessidades nutricionais do lactente ${ }^{(19)}$. Em Punjab, no Paquistão, as mães acreditam que o primeiro alimento oferecido a uma criança, após o parto, não deveria ser leite materno, mas mel, flor de rosa ou leite de cabra das mãos de um presbítero da família ou de uma pessoa religiosa ${ }^{(20)}$. Apesar de várias evidências sobre os benefícios do aleitamento materno exclusivo, muitas mulheres praticam o aleitamento misto, associando leite artificial. Outras informações devem ser incorporadas nas orientações em saúde, seja sobre o posicionamento que auxilia na amamentação ou no reflexo dessa prática sobre sono saudável do lactente (10,21-22). $^{\text {. }}$

Sobre os fatores impeditivos ao ato de amamentar e contribuintes ao desmame precoce, estão o retorno ao trabalho, dores mamilares, nas costas, na incisão cirúrgica, ingurgitamento, fadiga(23) leite insuficiente, crenças religiosas, 
rejeição à mama ${ }^{(10)}$ síndrome metabólica do bebê, excessiva carga de trabalho da mãe, falta de suporte social, bem como as estratégias de promoção e marketing da fórmula infantil(24). Esta pesquisa apontou dor, traumas mamilares e ingurgitamento como os três primeiros fatores relacionados ao desmame.

Neste estudo, houve associação entre a faixa etária e paridade com conhecimento, indicando prevalências maiores para adequabilidade dos saberes entre mulheres mais maduras e multíparas. Além disso, mulheres acima de 26 anos, retardo da iniciação sexual e multiparidade constituíram-se fatores de proteção ao conhecimento sobre ao aleitamento materno, ou seja, a multiparidade é indicada como fator de proteção para a decisão de amamentar exclusivamente.

Acredita-se que planejar uma família traz boas expectativas sobre a maternidade e 0 cuidado com a criança, melhorando as opiniões positivas sobre o AME. Essa crença pode ser corroborada pelos dados da pesquisa em Dakar, a qual identificou que $96,8 \%$ das mulheres multíparas amamentaram de maneira exclusiva $^{(25)}$.

Em Curitiba, histórico de paridade materna com amamentação anterior pode direcionar as próximas. Ao mesmo tempo em que as primíparas são mais propensas a iniciar o aleitamento, costumam mantê-lo por menos tempo, introduzindo alimentos complementares precocemente $^{(15) .}$ Há pesquisa que indique primigestas e multíparas com comum dificuldade relacionada ao reconhecimento do AM como alimento importante exclusivo nos primeiros seis meses de vida da criança ${ }^{(25)}$.

As mulheres fumantes amamentam menos. É importante destacar que o fumo causa alteração na composição do leite e diminuição da produção ${ }^{(20)}$. Embora haja escassez de pesquisas sobre a relação da etnia com o conhecimento insatisfatório sobre aleitamento materno, o conhecimento satisfatório pode estar relacionado a outros fatores, como formação superior, emprego remunerado e estabilidade da união, primiparidade ou multiparidade ${ }^{(13-14)}$.

Em relação ao suporte social, quanto maior o apoio das redes primária e secundária, maior a intenção das mães na adesão à amamentação ${ }^{(23)}$. Ainda sobre os dados de outros estudos, mulheres que vivem sem companheiros oferecem risco maior de amamentar seus bebês por menor tempo, quando comparadas às que possuem companheiro(13-15). Sobre CAP, há concordâncias na literatura sobre a satisfatoriedade do conhecimento e atitude ${ }^{(11,22-23)}$ com discordâncias sobre a prática(22).

Com efeito, acredita-se que a Enfermagem constitua uma parte integrante da rede de apoio secundária às mulheres, podendo fortalecer a prática satisfatória, a partir do cuidado técnicoafetivo no alojamento conjunto, bem como no retorno dessa mulher ao ambiente domiciliar, seja na visita puerperal, ou em consultas de puericultura na atenção básica. Portanto infere-se que o vínculo e o suporte da Enfermagem podem impactar positivamente o aleitamento materno, seja em âmbito hospitalar ou na contrarreferência da rede de atenção.

\section{CONCLUSÃO}

Os objetivos do estudo foram alcançados, permitindo indicar que, na amostra estudada, o conhecimento, a atitude e a prática foram satisfatórios, havendo vários fatores de risco e proteção a esses construtos. Além disso, dor, traumas mamilares, ausência da rede de apoio, ingurgitamento, mastite, leite insuficiente, falta de desejo de amamentar e complicação materna pós-parto foram elencados como motivos relacionados ao desmame precoce.

A avaliação, em um único hospital amigo da criança, impossibilita a generalização dos resultados e o delineamento transversal, o qual impede o acompanhamento dessas puérperas, ao longo do tempo, para verificar possíveis mudanças de concepções, constituíram-se limitações da pesquisa. Este estudo contribui com a comunidade científica, quando disponibiliza dados avaliativos relacionados aos serviços prestados em um Hospital Amigo da Criança, demonstrando que a certificação pode condizer com os esforços governamentais de fortalecimento do locus de pesquisa, enquanto instituição que protege, apoia e promove o aleitamento materno.

Além disso, os resultados e as reflexões geradas nesta pesquisa podem nortear os profissionais para desenvolver tecnologias em saúde (panfletos, manual e guias de aleitamento materno) para futuros treinamentos em hospitais similares. Os elementos de risco e proteção elucidados neste estudo possibilitam visualizar o perfil de mulheres que merecem um olhar mais atento às vulnerabilidades e lacunas do conhecimento, na intenção de facilitar a redução 
das fragilidades de opinião e favorecer o incentivo ao comportamento satisfatório frente ao aleitamento.

\section{REFERÊNCIAS}

1 - Aldrighi JD, Wall ML, Souza SRRK, Cancela FZV. The experiences of pregnant women at an advanced maternal age: An integrative review. Rev Esc Enferm USP 2016;50(3):512-21. DOI: 10.1590/50080-623420160000400019

2 - Paddock JD. Breastfeeding patterns among employees at a land grant university. Workplace Health Saf. 2017;65(12):580-94. DOI: 10.1177/ 2165079917701138

3 - Saco MC, Coca KP, Abrão ACFV, Marcacine KO, Abuchaim ESV. Contato pele a pele e mamada precoce: Fatores associados e influência no aleitamento materno exclusivo. Texto ContextoEnferm. 2019;28:e20180260. DOI: 1980-265XTCE-2018-0260

4 - Ferreira AP, Dantas JC, Souza FMLC, Rodrigues IDCV, Davim RMB, Silva RAR. O enfermeiro educador no puerpério imediato em alojamento conjunto na perspectiva de Peplau. Rev Eletr Enf. 2018;20:1-9. DOI: 10. 5216/ree.v20.45470

5 - McCardel RE, Padilla HM. Assessing Workplace Breastfeeding Support Among Working Mothers in the United States. Workplace Health Saf. 2020;68(4):182-9. DOI: _ 10.1177/2165 $\underline{079919890358}$

6 - Andrade SSDC, Zaccara AAL, Leite KNS, Brito KKGD, Soares MJGO, Costa MML, et al. Knowledge, attitude and practice of condom use by women of an impoverished urban area. Rev Esc Enferm USP 2015;49(3):364-71. DOI: S0080-623 420150000300002

7 - Ghisi GLM, Durieux A, Manfroi WC, Herdy AH, Carvalho $T$, Andrade $A$, et al. Construction and validation of the CADE-Q for patient education in cardiac rehabilitation programs. Arq Bras Cardiol. 2010;94(6):763-71. DOI: $10.1590 \quad /$ s0066$\underline{782 \times 2010005000045}$

8-Olsen JM, Lago TDG, Kalckmann S, Alves MCGP, Escuder MML. Young women's contraceptive practices: A household survey in the city of São Paulo, Brazil. Cad Saúde Pública 2018;34(2):e0001 9617. DOI: 10.1590/0 102$\underline{311 \times 00019617}$
9 - Victora CG, Bahl R, Barros AJ, França GV, Horton S, Krasevec J, et al. Breastfeeding in the 21st century: Epidemiology, mechanisms, and lifelong effect. Lancet 2016;387(10017):475-90. DOI: 10.1016/S0140-6736(15)01024-7

10 - Thet MM, Khaing EE, Diamond-Smith $N$, Sudhinaraset $M$, Oo $S$, Aung $T$. Barriers to exclusive breastfeeding in the Ayeyarwaddy Region in Myanmar: Qualitative findings from mothers, grandmothers, and husbands. Appetite 2016;96(1):62-9. DOI: 10.1016/ j.appet.2015.08.044

11 - Viduedo AFS, Leite JRC, Monteiro JCS, Reis MCG, Gomes-Sponholz FA. Severe lactational mastitis: Particularities from admission. Rev Bras Enferm. 2015;68(6):806-11. DOI: 10.1590/0034$\underline{7167.2015680617 i}$

12 - Santos LAV, Lara MO, Lima RCR, Rocha AF, Rocha EM, Glória JCR, et al. Gestational history and prenatal care characteristics of adolescent and adult mothers in a maternity hospital in the interior of Minas Gerais, Brazil. Ciên Saúde Colet. 2018;23(6):617-25. DOI: 10.1590/141381232018232.10962016

13- Cardoso AMR, Marín HF. Gaps in the knowledge and skills of Portuguese mothers associated with newborn health care. Rev LatinoAm Enfermagem 2018;26:e2997. DOI: $\underline{10.1590 / 1518-8345.1859 .2997}$

14 - Al Ketbi MI, Al Noman S, Al Ali A, Darwish E, Al Fahim M, Rajah J. Knowledge, attitudes, and practices of breastfeeding among women visiting primary healthcare clinics on the island of Abu Dhabi, United Arab Emirates. Int Breastfeed J. 2018;13(1):26. DOI: 10.1186/s13006-018-0165

15 - Martins FDP, Leal LP, Linhares FMP, Santos AHDS, Leite GDO, Pontes CM. Effect of the board game as educational technology on schoolchildren's knowledge on breastfeeding. Rev Latino-Am Enfermagem 2018;26:e3049. DOI: $\underline{10.1590 / 1518-8345.2316 .3049}$

16- Gejo NG, Weldearegay HG, Tinsaie KT, Mekango DE, Woldemichael ES, Buda AS, et al. Aleitamento materno exclusivo e fatores associados entre mães soropositivas no norte da Etiópia. PLoS One 2019;14(1):e0210782. DOI: 10.1371/journal.pone.0210782 
17- Osibogun OO, Olufunlayo TF, Oyibo SO. Knowledge, attitude and support for exclusive breastfeeding among bankers in Mainland Local Government in Lagos State, Nigeria. Int Breastfeed J. 2018;13(1):38. DOI: 10.1186/s 13006-018-0182-9

18- Gewa CA, Chepkemboi J. Maternal knowledge, outcome expectancies and normative beliefs as determinants of cessation of exclusive breastfeeding: A cross-sectional study in rural Kenya. BMC Public Health 2016;16(243):1-9. DOI: 10.1186/s12889-016-2907-2

19-Mogre V, Dery M, Gaa PK. Knowledge, attitudes and determinants of exclusive breastfeeding practice among Ghanaian rural lactating mothers. Int Breastfeed J. 2016;11(12):18. DOI: $10.1186 / s 13006-016-0071-z$

20 - Zakar R, Zakar MZ, Zaheer L, Fischer F. Exploring parental perceptions and knowledge regarding breastfeeding practices in Rajanpur, Punjab Province, Pakistan. Int Breastfeed J. 2018;13(1):24. DOI: 10.1186/s13006-018-0171-z

21 - Gupta PM, Perrine CG, Chen J, Elam-Evans LD, Flores-Ayala R. Monitoring the World Health Organization global target 2025 for exclusive breastfeeding: Experience from the United States. J Hum Lact. 2017;33(3):578-81. DOI: $\underline{10.1177 / 0890334417693210}$

22 - Boccolini CS, Boccolini PDMM, Monteiro FR, Venâncio SI, Giugliani ERJ. Breastfeeding indicators trends in Brazil for three decades. Rev Saúde Pública 2017;51:108. DOI: $\underline{10.11606 / S 1518-8787.2017051000029}$

23 - Senghore T, Omotosho TA, Ceesay O, Williams DCH. Predictors of exclusive breastfeeding knowledge and intention to or practice of exclusive breastfeeding among antenatal and postnatal women receiving routine care: a cross-sectional study. Int Breastfeed J. 2018;13(1):9. DOI: 10.1186/s13006-018-0154-0

24 - Cascone D, Tomassoni D, Napolitano F, Di Giuseppe G. Evaluation of knowledge, attitudes, and practices about exclusive breastfeeding among women in Italy. Int J Environ Res Public Health 2019;16(12):2118. DOI: 10.3390/ijerp $\underline{\text { h16122118 }}$

25 - Gueye M, Mbaye M, Diallo M. Knowledge, attitudes and practices of mothers on breastfeeding in urban environment in dakar. MOJ Womens Health 2018;7(3):83-7. DOI: $\underline{0.15406 /}$ $\underline{\text { mojwh.2018.07.00175 }}$

\section{Editores Responsáveis:}

Juliana Dias Reis Pessalácia

Gisele Nepomuceno de Andrade

Nota: Pesquisa originada de um Trabalho de Conclusão de Curso apresentando a Faculdade de Enfermagem Nova Esperança para aquisição do título de bacharel em Enfermagem.

Recebido em: $17 / 12 / 2020$

Aprovado em: 25/05/2021 